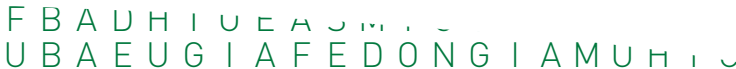

$N L|B F| M B C H S E H E B P S K R B E F B A r$.

$S E H W C E C B S T P O \mid O D V F E D B E U B A F \vee N K$,

$P O|F E G \vee T Q U J X R E L J H G G E \cup N L| E R N G M J b$,

J Z R A N L M R TXAGYWUF I M B C H S E H B U P S K S A U M

A G Y F T XVNHOUB I JECEfrinaT P O I O D C V F E FEQL O ,

U B I R R R TFLU JAD J J RELKJHGECLZEMS.

JADVIDBPORU

UTEEFCSKUP

$L K J R G D X A Y$ A S GW A ZUKC I KCGLUCZ $M Q G Z D A T S$ $K Z$ I W P LOM S C V NEAMU $J Y Q G N M N S$ O JNP D | EP $R A \cup F U S A T$ $P D B A L I E P$ $R A \cup G \cup \cup C Z$ $M O Q F M S A T B$ $P D B F-G \vee T Q$ O T R J T D S Y K $\begin{array}{lllllllll}Y & A & Z & J & Y & C & K & O & I \\ W & C & L & T & P & Q & O & G & N\end{array}$ NFX XEINRLU H GKLOZVCESOP NO | | A Q Y A H | N d U F B A K B N U R A K D O

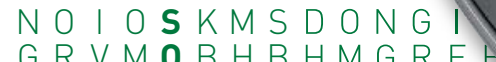
GR VMOBHBHMGRE E $F B A O R \cup G N D O N G \mid \cup A$ F D V I B R A T I O N N S Q O O E LEMEBZGVTFCRDXBDLRBEFDAFVNKFNKREWBPLOCYQDMFEF FCR I R Y C B MWRZ I P S Q LKJHGFDSAMMBVCXYMLSOKN I JBHUZGF $R Z$ I E UMBCYNVXADGW IVBAUELEMENTERFVEGOZHNUJMIKOQA VXAMKIJHLMOKN I JHBZGVTFCRDXESNWASRERVFHKNUTEQTF EDKTJDGLETUOADGLYCBMWRZ I PSFHKTVNZLBO I JEUHBZGWR REWZUETOIZRWQETOMBCYNVXADGJLKHESYSEBFGMHTILQNV 


\section{Isolation is the Key}

\section{The evolution of the centrifugal \\ pendulum-type absorber not only for DMF}

Dr. Ad Kooy

D v

$J \mathrm{ZMH}_{L}$

$A G Q S W \mid\llcorner\cdots$

I I I B CHSEHE七

C E C B S T P O I O D C V ז

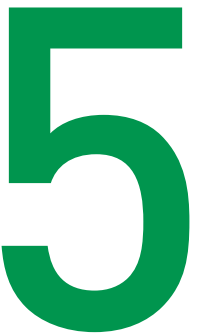

$D G \vee T Q U J X R E L K J H G F D S A \mid V_{1} \cdots$

YLMRTXAGYWPHCEQA YWS XE E C K ,

I X X V N OUB I JBZGVTFCRDXESNWASKL

$Z \vee T F L U J A D G Y C B M W R Z$ I P S F H K T VNZLMU,

$X D B P O R U T E T M B C Y N V X A D G J L K H E S Y S C B M B \smile$

DC SKUP OWRWZTWHNEDKUNWPONCALVIKZTWHIN

EHKLPFLKJKO I UZTREWQYXCVBNM I QWUO I UZTR

WDXAYHASGSVNPIZRWQSCGZNJIMNSTRVNPIZRWQ_

$L Z U K O G I K C K P M N E S W L N C X W Z Y K F E D I O P P M N E S W L N C X$

I $R \cup C Z G Z M Q G O D N V U S G R V L G R V K G E C E Z E M D N V U S G R V L G h$ QATSLOKZINEXOMNYAZTEWNFX JLRNIFEXOMNYAZTEW $C L O M E P S C V C Y I, \ldots, \cdots \cdots 1, V \vee F H N R D J K U V Y L J N E W C L \vee V F$ FAMUANJYC'. 'SQFHBQFGOBRELNFXTJC KMNSRDO _ I IV U L I ZPMFDROIDFNGKLDFN
LI E P N N

B S A T B

$P \mid$ E P P

$R \cup C Z$

B S A

D G V

$Y\llcorner\wedge$

$C \times$

Z V

$X \mathrm{D}$
, I I O G G D O I EK,

$D D L R B E F B A V N K$

. UAH | OGDNO | ERNGM

OQ ODNVUSGR V L GR VKG

$P D B D D L B E F B A F V N K F N$ r

, OTRELKJHGFDSAMMBVCX $X A Z Y W P H C E Q A Y W S X E E C R F$ I $O U B$ I JBZGVTFCRDXESNWAS U JRDGYCBMWRZ I PSFHKTVN RUTETMBCYNVXADG JLKHES P OWRWZTWHNEDKUNWPONCA FLKJKO I UZTREWQYXCVBNM HASESVNPIZRWQSCGZNJ|M G I KCKPMNESWLNCXWZYKFF

I A S U S VNP I ZRWQSCGZN J I

I KCKPMNESWLNCXWZYKF

' OXODNVUSGRVLGRVKG

1 I NEXOMNYAZTEWN F

' CYL JNEWCLVVFH'

$\cap N \vee \cup S G R \vee L G F$
ऽ $A \cup K Z Q H|O G D N O| E R N$ I

PEWS PDLRBEFBAFVNK

LKZQH IOGDNO।ERNGN Z EMDNVUSGRVLGRVK NSPDLRBEFBAFVNKF $M O L K J H G F D S A M M B$ B Z P HCEQAYWSXEEC $\approx V B Z G V T F C R D X E S N$ 10 Y C B MWR Z I P S F H K

BMBCYNVXADGJLK KZTWHNEDKUNWPr 


\section{Introduction}

A key task that has concerned the automotive industry in recent years has been to reduce consumption. One effective measure for achieving this goal is to exploit even lower engine speeds for driving. Torque is increased to achieve this without losing power. Doing so allows the engine to run only very slightly above idle speed and therefore in an extremely consumptionefficient range. One challenge is to achieve adequate powertrain isolation even for these low engine speeds and thus provide drivers with their usual level of comfort.

Figure 1 [1] shows that the dual mass flywheel (DMF) is a factor in achieving this goal, particularly in connection with the centrifugal pendulum-type absorber. While twin-cylinder engines have yet been unable to reach the projected fuel savings for day-to-day use, the increasing numbers of three-cylinder engines have achieved lower consumption figures. However, lower consumption places stricter demands on vibration isolation. The secondary-side centrifugal pendulum-type absorber (CPA)

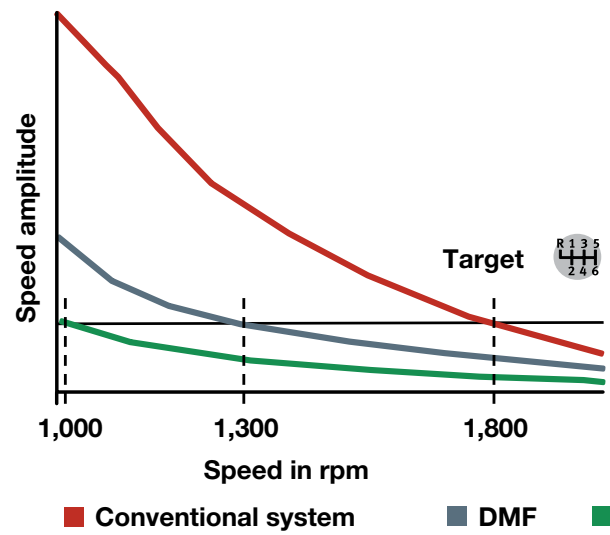

was introduced as a concept in conjunction with the DMF as early as 2002 [2], and successfully went into series production a few years later. The simple physical principle, modular design and extremely good isolation have led to increasing acceptance and proliferation not only in the DMF, but also in other damping concepts such as torque converters and clutch discs. There have also been huge improvements in how the centrifugal pendulum-type absorber works thanks to far-reaching understanding of the centrifugal pendulum-type absorber; more detailed information is provided about this below.

As the DMF must also be optimised for other operating points, such as startup, or optimised for so called impacts - very high torque peaks when bottoming out the arc springs - compromises must be made. These compromises also have an indirect influence on isolation in drive mode. We will be using examples of impacts that affect DMFs when stalling the vehicle and demonstrating methods of preventing stress of this kind and making DMFs more robust. These result in greater freedom for optimising torsion isolation and so improving driving comfort.

\section{$\mathrm{l} / 100 \mathrm{~km}$}

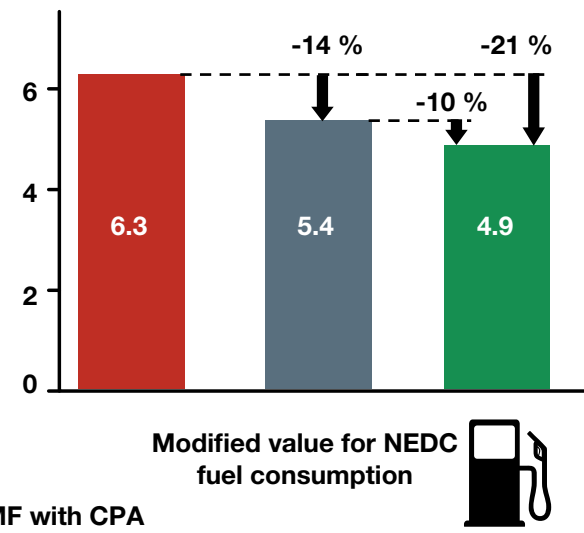

Figure 1 Fuel economy potential with DMF and DMF with CPA

Schaeffler Technologies GmbH \& Co. KG, Solving the Powertrain Puzzle,

DOI 10.1007/978-3-658-06430-3_5, (c) The Author(s) 2014 


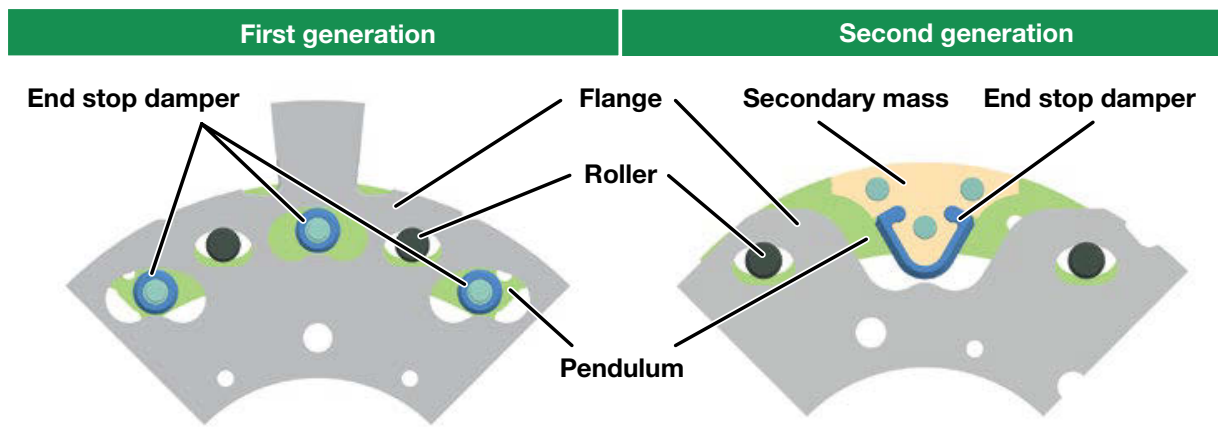

Figure 2 Layout of end stop dampers on first and second-generation CPAs

\section{Development of DMF centrifugal pendulum-type absorbers}

To date, one million centrifugal pendulumtype absorbers have been produced for sixcylinder, four-cylinder and three-cylinder engines, and the concept has been continually developed. Prototypes show that the technology could also be employed in twincylinder engines.

The secondary-side arrangement of the centrifugal pendulum-type absorber makes the arc spring damper, which provides preisolation, especially important. Taking engine torque development into consideration largely automated simulation programs run through hundreds of variations evaluating start and drive to find the optimum combination of arc spring and CPA for a vehicle application. Of course, this requires vehicle parameters of adequate quality which are not always available during the early stages of development in which design takes place. This is where LuKs wealth of experience really comes into its own, as it allows us to complete missing data in a meaningful manner. However, should corrections be required subsequently during to vehicle test- ing, simulations of this kind can quickly be repeated. Interaction with other critical operating points can also be integrated, such as stalling the engine along the critical details of engine timing management.

It is easy to calculate the natural frequency of a thread pendulum, in other words a point mass moving on a circular path, if the angle is small. However, this approach is inadequate for centrifugal pendulum-type absorbers. The path curvature must be more pronounced to maintain a constant order (natural frequency to speed frequency ratio) independently of the magnitude of the angle. This approach is the only way to achieve optimum isolation over the whole engine speed for partial throttle as well as for wide-open throttle. Special attention must be given to the rpm range slightly above idle speed. On account of the low centrifugal forces in this range, the CPA needs as large a vibration angle as possible to store sufficient vibration energy. High engine torques exacerbate the situation. Therefore, the goal is to maximise this angle along with the pendulum inertia. For this reason, the three circular end stop dampers previously present on first-generation centrifugal pendulum-type absorbers have been combined into a $\mathrm{V}$ shaped end stop damper on an additional intermediate mass in second-generation absorbers (Figure 2). 
This eliminates the need for the beanshaped holes in the flange required for the circular end stop dampers and creates additional space for greater vibration angles or heavier pendulums. The added intermediate mass lies relatively far towards the outer edge in radial terms, thereby improving isolation in the low speed range through increased inertia. A number of other optimisations, such as optimising the arc spring damper with the centrifugal pendulum-type absorber as a system, smoother pitch surfaces and optimised paths, have together resulted in a significant performance boost, especially at low engine speeds (Figure 3).

The example of a four-cylinder diesel engine shows that when using the first generation absorber an increasing of the engine torque from 360 to $450 \mathrm{Nm}$ leads to a clear deterioration in isolation. In contrast, when the second generation is used, a torque in- crease can be handled without loss of comfort. For three-cylinder engines acceptable values of $500 \mathrm{rad} / \mathrm{s}^{2}$ from about 1,000 rpm are already achieved (in this example, a diesel engine with $270 \mathrm{Nm}$ ). However, these values can still be significantly reduced: If the entire clutch system - i.e. DMF with centrifugal pendulum-type absorber and clutch - is designed according to an entirely new layout, (third generation), it is possible to achieve angular acceleration amplitudes of below $200 \mathrm{rad} / \mathrm{s}^{2}$ from $800 \mathrm{rpm}$ upwards and without requiring any further space. The rigidity of drive shafts, in particular, must be incorporated into this concept. If rigidity changes, it results in a completely new design. It makes close coordination with the vehicle manufacturer's development process essential.

The considerations mentioned above relate to a centrifugal pendulum-type absorber integrated below the arc spring

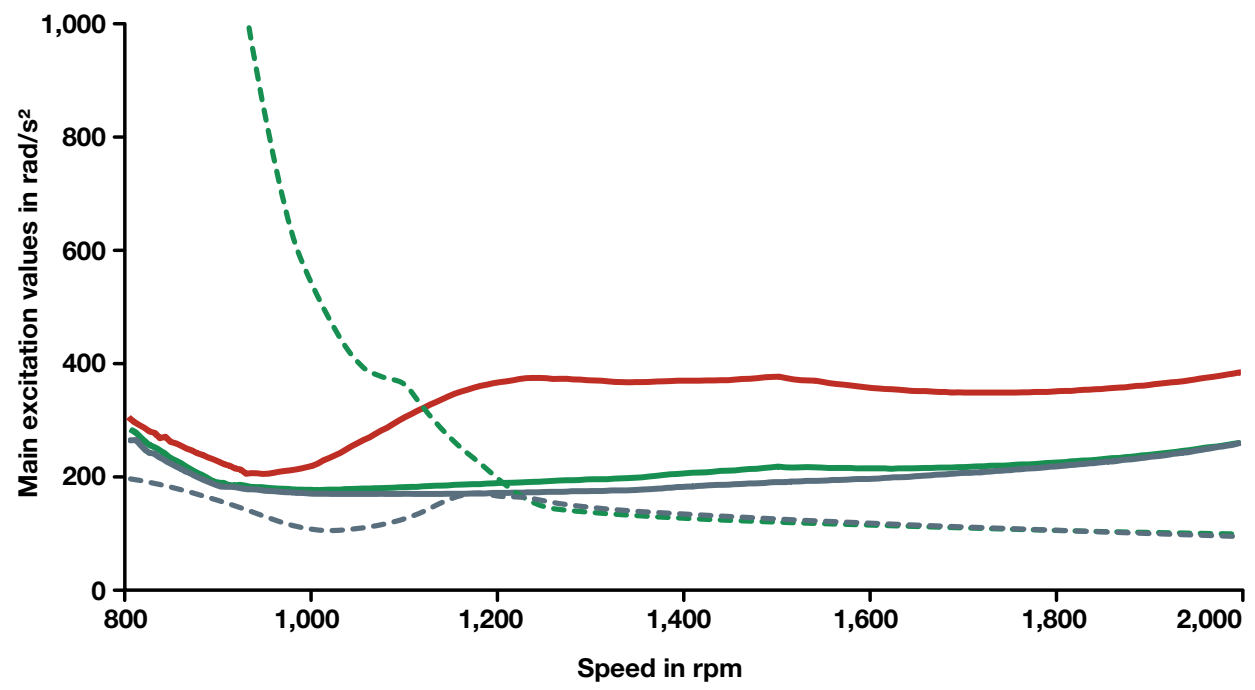

$$
\text { Four-cylinder } \begin{aligned}
- & 450 \mathrm{Nm}-\text { generation } 1 \\
& -450 \mathrm{Nm}-\text { generation } 2 \\
& -360 \mathrm{Nm}-\text { generation } 1
\end{aligned}
$$

Three-cylinder -- $270 \mathrm{Nm}$ - generation 1

-- $270 \mathrm{Nm}$ - generation 3

Figure 3 Comparing DMF isolation with various CPA generations in three-cylinder and four-cylinder engines 


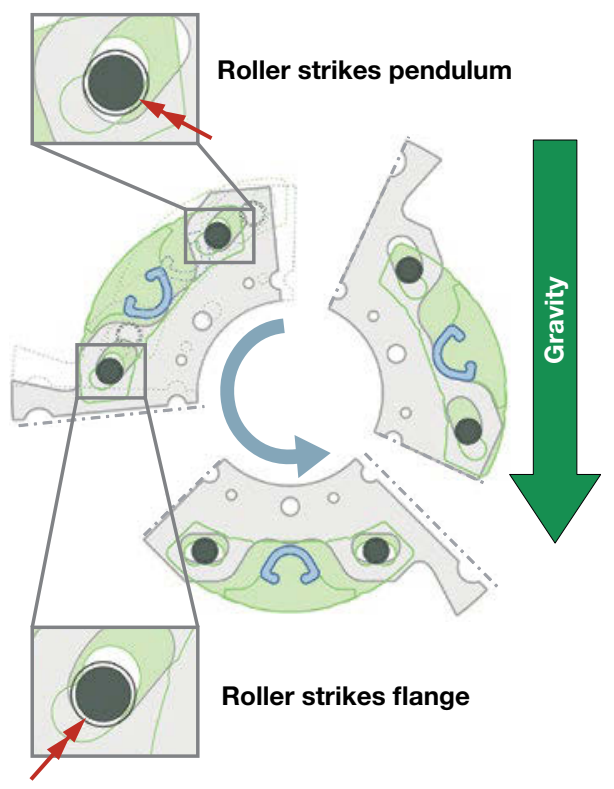

Figure 4 Kinematic simulation of pendulum motion at $150 \mathrm{rpm}$

damper. As already shown in [1], a centrifugal pendulum-type absorber can also be arranged next to the arc spring, i.e. radially further towards the edge, if sufficient space is available; this improves isolation even further, where necessary. For engines without cylinder deactivation, as commonly used in series production, it is therefore possible to achieve adequate isolation using a centrifugal pendulum-type absorber. Should a CPA of this kind provide isolation better than that required, costs can be reduced by omitting two of the four pendulum masses.
Path wear is not expected due to the fact that the pendulum only has a rolling motion. However rattling noises may occur when switching the engine off: As soon as the engine speed drops below approx. $200 \mathrm{rpm}$, the pendulum's centrifugal force drops below the force of gravity. It falls a few millimetres within the designed degree of freedom until it strikes the bolts on the flange. In order to better understand this process, kinematic simulations have been carried out and compared using high-speed recordings (Figure 4).

The simulation demonstrates how the two rollers strike differently; the precise arrangement of the damper and rollers and the clearance between the roller components have an effect on these striking patterns. These parameters must be precisely analysed and optimised. In addition to these kinds of optimisations, ways of preventing stopping noises have also been investigated. One option is to arrange circular end stop dampers at the end of the pendulum (Figure 5).

This causes the pendulums to strike each other after a short fall, and a part of the kinetic impact energy stored in the pendulum system is neutralised without any noise occurring. The rollers striking on the

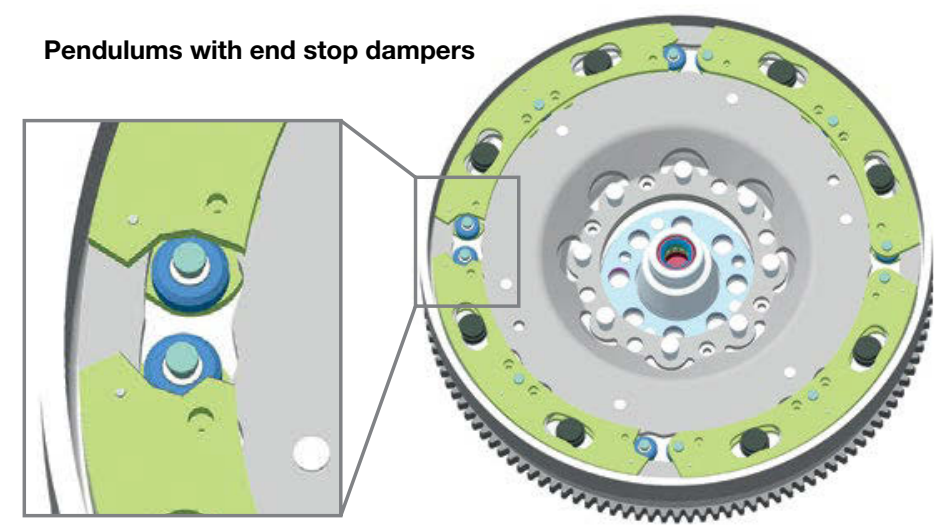

Figure 5 CPA on outer edge with end stop dampers between the pendulums 
flange only cause slight noise. This concept works well with a closed throttle valve, as pure torsional acceleration is low in this state. However, much higher torsional acceleration occurs when stopping if the throttle valve is to remain open, for instance to enable cylinders to charge correctly to enable quick automatic start-up. The result is that all pendulums have a virtually synchronised torsional motion, thereby rendering the rubber stops on the end of the pendulum ineffective; their job is then assumed by the central $\mathrm{V}$-shaped end stop damper.

\section{Centrifugal pendulum- type absorber mounted on the clutch disc}

The success of the DMF is due to the fact that hypercritical operation is largely possible, compared to torsion-damped clutch discs. The result is an enormous increase in isolation, as already shown in an example in [3]. Also discussed in [3] was the option of arranging a centrifugal pendulum-type absorber on the clutch disc - positioned on the gearbox input shaft for simulation purposes. Based on the knowledge of pendulum path design, permissible mass moments of inertia and tolerances permitted in series production available at that time, a viable solution was not within reach. Today, our in-depth expertise concerning the design of centrifugal pendulum-type absorbers coupled with new ideas on the reduction of clutch disc mass inertia means this approach can be implemented (Figure 6).

For clutch discs with a single pendulum system, it comprises of two or three pendulums and is calibrated to the main excitation, i.e. order 1.5 for a three-cylinder engine. Clutch discs with double pendulum systems have two additional auxiliary pendulums, calibrated to double the main excitation frequency. In both designs, the pendulums are arranged next to the damper. During development, a particular aim was to keep the extra clutch disc inertia caused by
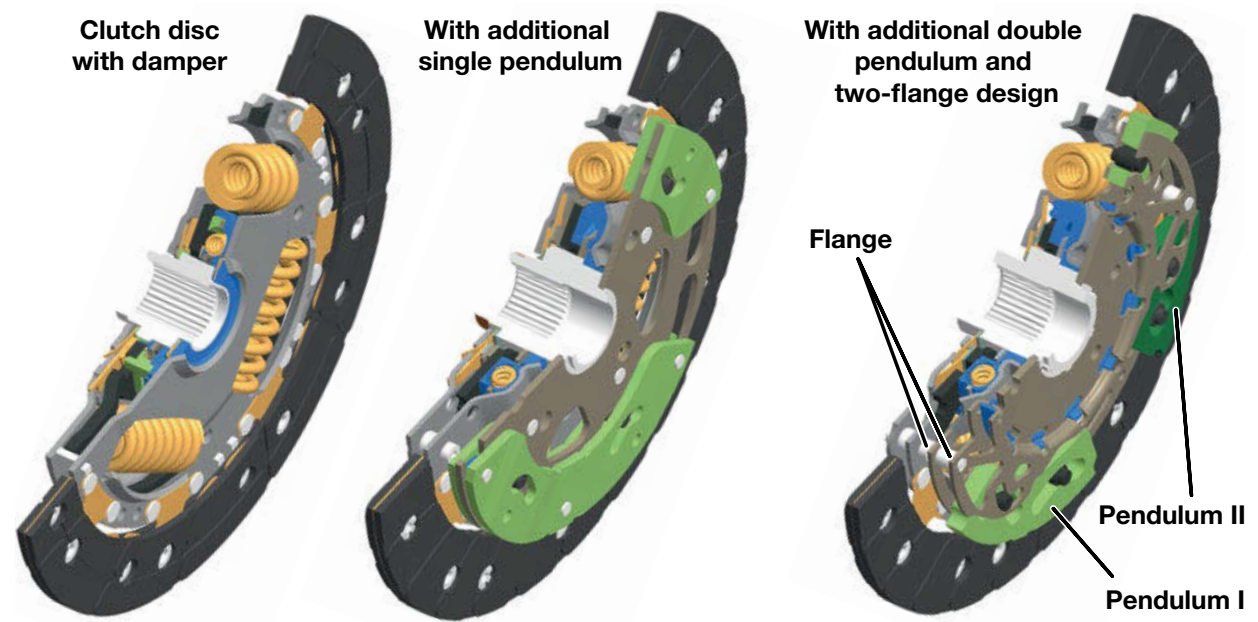

Figure 6 Clutch discs without a pendulum, with a single pendulum and with a double pendulum system 
the pendulums to a minimum, so that gear synchronisation was not overloaded. Therefore, the pendulums needed to be particularly effective despite their low mass. As the effect of a pendulum is mainly determined by the product of mass and vibration angle, the vibration angles consequently had to be hugely enlarged.

Initial designs for the first generation used three pendulums. In the optimised, second-generation version, two pendulums with secondary spring masses were used for clutch discs with a single pendulum system (Figure 7).

The additional intermediate mass was introduced along the same lines as the DMF (Figure 2): Therefore, more mass can be arranged on the outer edge in radial terms. But the most important innovation concerns the two roller paths of each pendulum. The paths are now no longer identical and are now skewed relative to one another instead of merely displaced. This is reflected in the skewed arrangement of the bean-shaped holes for the rollers in the pendulums, as a comparison of the first and second generations shows. This arrangement causes the pendulum to execute a rotation in addition to oscillation. The sketch in Figure 7 illustrates this principle: During movement, the end of the pendulum is guided radially inwards while the other end simultaneously moves radially outwards. This arrangement has become known as a trapezoidal pendulum, while the first generation is called a parallel pendulum.

Thanks to their trapezoidal oscillation, the pendulums need less space meaning that considerably larger pendulum vibration angles can be achieved. Additional rotational energy is also stored when turning, so better use is made of the pendulum mass.

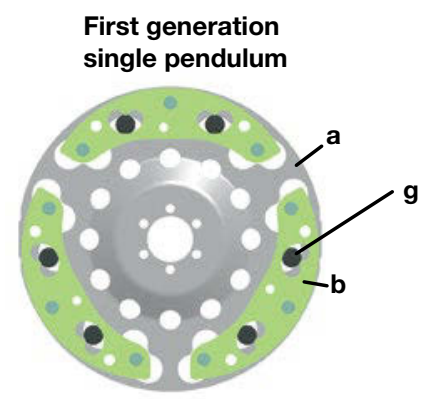
a) Flange
b) Main pendulum
c) Secondary pendulum
d) Intermediate mass
e) End stop damper
f) Pressure spring
g) Roller

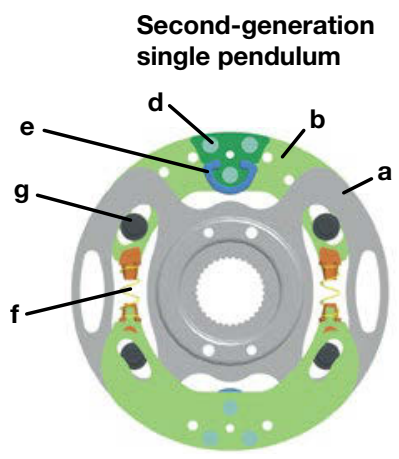

Second-generation double pendulum

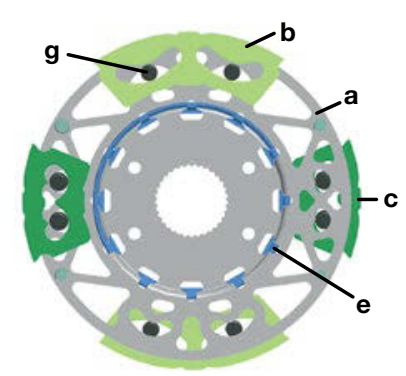

Trapezoidal pendulum (second generation)

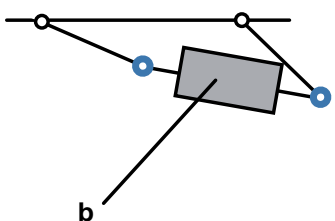

Figure 7 CPA for clutch discs 
This effect can also be utilised on the DMF, but it is not so effective there due to the mounting space available.

Although the pendulum masses are lower than those of the DMF, undesirable knocking noises may occur when stopping if the bell housings are sensitive or open. The spring bracing of second-generation pendulum masses (Figure 7) also helps combat this problem. The preloaded springs can be designed to be especially soft thanks to the reduced pendulum masses. This is important because the spring forces are not speed-dependent and do not follow the principle of the centrifugal pendulum-type absorber. An angular correction of path geometry minimises this effect.

Figure 8 shows a comparison of a DMF with a single mass flywheel with CPA on the clutch disc and a torsion-dampened clutch disc using the example of a four-cylinder engine. The single mass flywheel with a CPA on the torsion-dampened clutch disc takes

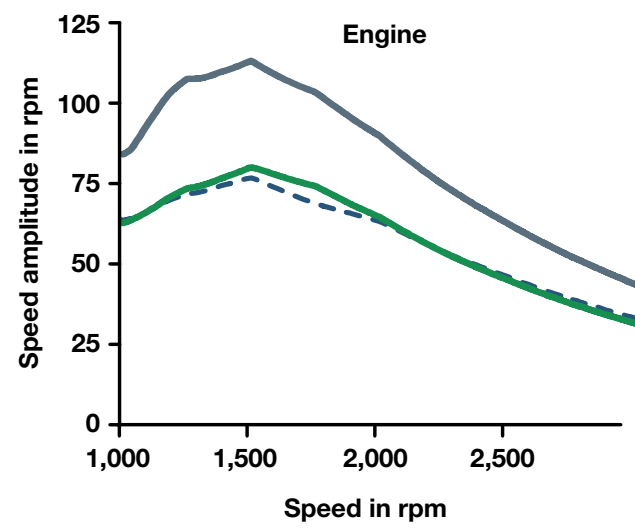

- Torsion-damped clutch disc + CPA clutch disc
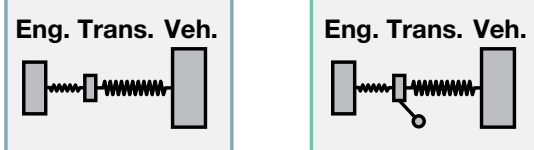

the middle position with regard to isolation of the torsional vibrations from the gearbox. On the engine side it even leads to smaller irregularities than a DMF, resulting in a lower load on the belt drive. This configuration proves its worth for three-cylinder engines in conjunction with soft drive shafts. However, when combined with rigid shafts, we have the problem that the third order comes through very dominantly in the overall amplitude of gearbox acceleration (Figure 9). The figure shows the total amplitude in which both orders arrive.

To dampen the third order, an additional pendulum system calibrated to this order has to be added; in other words, a double pendulum system is required. Figure 6 and 7 show the layout of both pendulums on the clutch disc. It goes without saying that only smaller pendulum masses are possible due to space constraints, but this is compensated in part by a dual-flange design. In this design, the pendulum is situated between

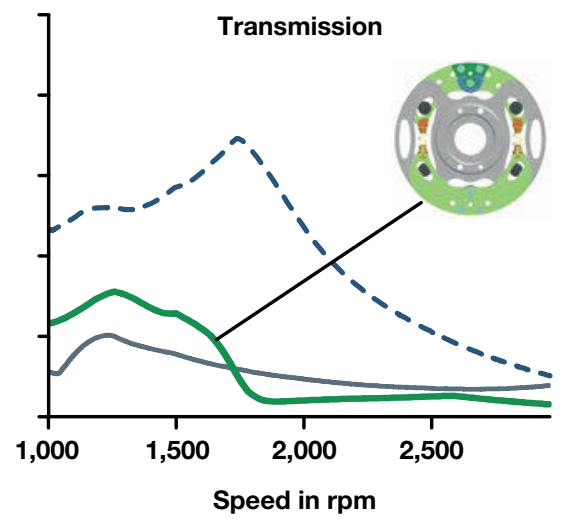

-- DMF

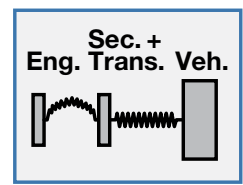

Figure 8 Comparing three damping concepts based on isolation of a four-cylinder engine in $6^{\text {th }}$ gear 


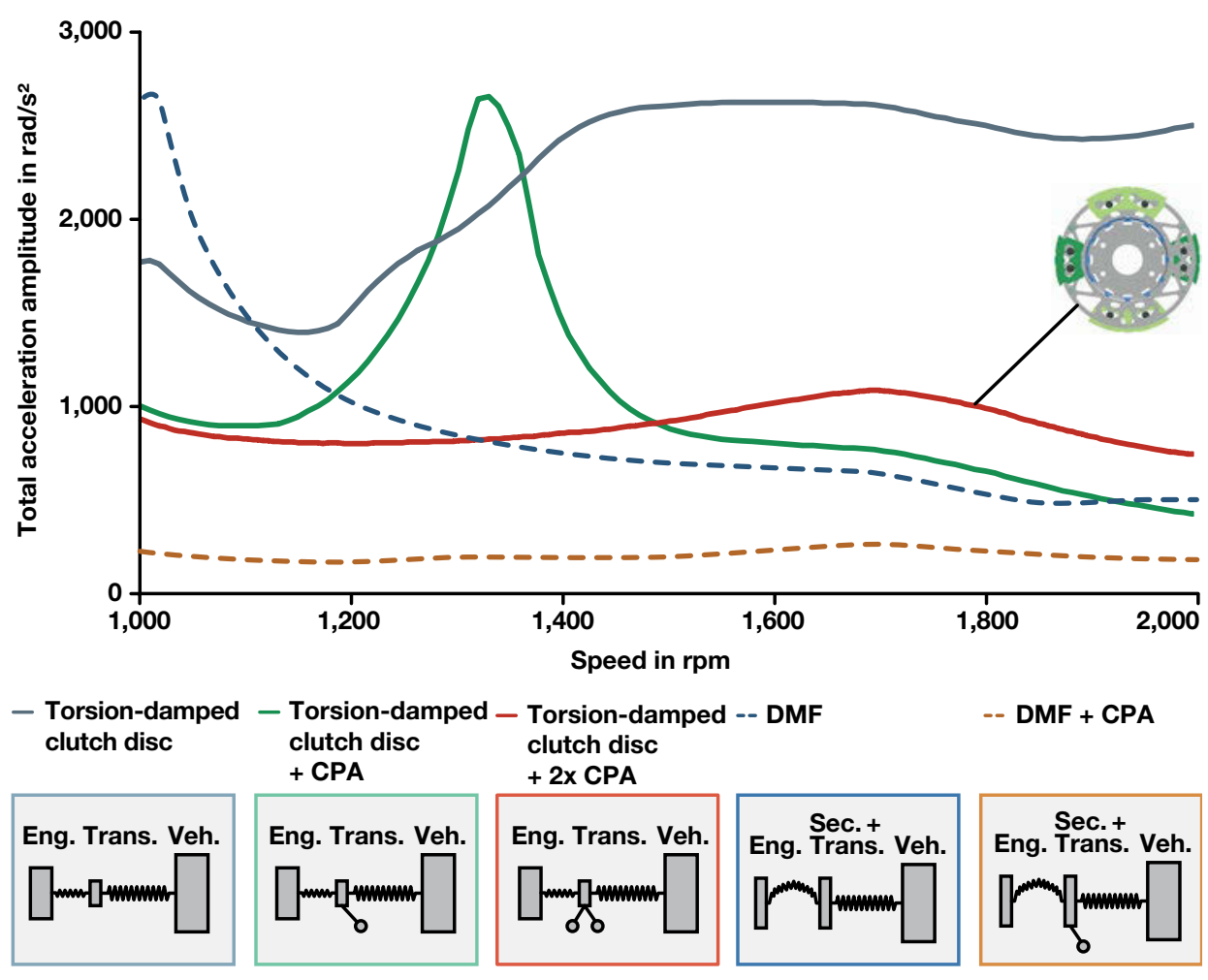

Figure 9 Comparing five damper concepts based on isolation of a three-cylinder engine with rigid side shafts in $6^{\text {th }}$ gear

two flanges. In the contrary, on a DMF it is usual practice for two pendulums to be arranged around a central flange (Flgure 2). This new design principle omits the connection elements of the sub-pendulums, which weaken the flange. As a result, larger pendulum vibration angles can be integrated. The achievable isolation reveals astonishing results: in $6^{\text {th }}$ gear, isolation below $1,300 \mathrm{rpm}$ is even better than with a DMF. However, if the DMF is combined with a centrifugal pendulum-type absorb$\mathrm{er}$, it is once again clearly the superior combination.

In order not to place additional stress on gear synchronisation, the entire mass inertia must not be significantly greater than for a normal torsion-dampened clutch disc, despite the CPA. This is achieved by reducing the mass of all individual parts affected. Detailed comments about mass reduction of this kind can be found in another article [4]. In conjunction with a CPA, the actual torsional damper in the clutch disc is dampened to a lesser extent which benefits isolation at higher engine speeds. Another significant benefit is that the centrifugal pendulum-type absorber aids isolation in the creeping range, i.e. the low torque range. This allows the creeping stage to be designed for steeper rates and higher torques. In this way, creeping rattle can be largely prevented.

The introduction of clutch discs with centrifugal pendulum-type absorbers pro- 
vides additional damping solutions, depending on vehicle configuration and the required isolation level. Simulations help when it comes to selecting the optimum damping parameters whilst taking complex boundary conditions into account. They can be used to implement a solution halfway between a DMF and a torsion-damped clutch disc both in terms of isolation and costs and long-awaited by the automotive industry.

\section{Centrifugal pendulum-type absorbers for trucks}

In comparison to passenger cars, significant damping of a truck gearbox requires considerably higher inertia of the centrifugal pendulum-type absorber on the clutch disc. However, this higher inertia leads to an unacceptable reduction of synchronisation service life, which at $1,000,000 \mathrm{~km}$ is well above the requirements for passenger cars. For this reason, other ways of improving isolation have been explored: The CPA was arranged on the single mass flywheel (Figure 10). It can be detached for easy maintenance.

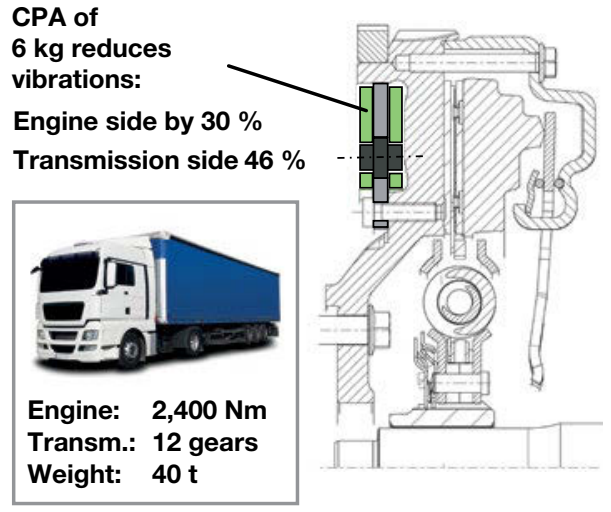

Figure 10 CPA on a truck single mass flywheel

The solid pendulums, which weigh around $6 \mathrm{~kg}$, reduce engine vibrations by $30 \%$ for a typical six-cylinder engine at 2,400 Nm, and reduce gear vibrations by $46 \%$. The latter directly improves the gearbox service life, as it is restricted if the vehicle is often driven at low engine speeds. In contrast, using a single mass flywheel with CPA can reduce engine speed without compromising service life when only low to medium engine torques are used, as is often the case (Figure 11). Fuel consumption is reduced by $5 \%$, which represents a competitive edge for end customers that should not be underestimated. The service life of the belt drive also benefits from reduced engine vibrations, thereby allowing this drive to be more simply constructed or service intervals to be extended.

Figure 11 Fuel consumption savings in a truck thanks to a single mass flywheel with CPA 


\begin{tabular}{|l|c|c|c|}
\hline Points of operation & Impact level & $\begin{array}{l}\text { Frequency of } \\
\text { occurrence }\end{array}$ & Meaning \\
\hline Stalling when moving off & High & Medium & \\
\hline Misshift $\mathbf{2}^{\text {nd }}$ to 5 $^{\text {th }}$ & High & Rare & \\
\hline Fast clutch engagement & Medium & Rare & \\
\hline Back shifting in while using throttle & Medium & Infrequent \\
\hline Engine start & Medium & Infrequent \\
\hline Emergency braking & Medium & Rare & \\
\hline Jackrabbit start & Low & Rare & \\
\hline
\end{tabular}

Figure 12 Classifying impacts

\section{Reducing impacts}

The principle of a DMF (without centrifugal pendulum-type absorber) is ultimately based on shifting the resonance speed of the powertrain from the driveable range into ranges well below idle speed. By shifting this speed, hypercritical driving is possible throughout the entire speed range with the resulting excellent isolation. Even in the early days of DMF development, it became clear that driving situations below idle speed, such as that occur when stalling a vehicle, lead to large vibration angles and the DMF can strike the end stops (impact). The energetic transition of high kinetic energy in the relatively rigid end stop results in torques that can be up to 40 times the engine torque. Impacts can also occur at other operating points, however not usually at this level or with this regularity (Figure 12).

Many ideas for reducing impacts have already been developed and implemented. The majority of them actually contradict the primary task of the damper system, i.e. isolation, by requiring additional mounting space (such as a slipping clutch in the flange) or using thicker (more robust) spring wires (damping arc springs). The following describes one approach using software and one using hardware; these approaches dramatically cut the severity and regularity of these kinds of impacts.

\section{Influence of the engine control unit when stalling}

Figure 13 shows a typical stalling measurement of a three-cylinder diesel engine plotted in an engine speed/time diagram. It can be seen that powerful impacts are caused by the extreme difference in speed between the primary and secondary side. To aid understanding, this diagram is converted into speed squared $\left(n^{2}\right)$ over crankshaft angle; this is because combustion causes an injection quantity to be turned into kinetic energy, which, in turn, is proportional to $\mathrm{n}^{2}$. Thus, cyclic engine irregularities for the same injection quantity are shown as the same amplitudes regardless of engine speed. It is appropriate to use the crankshaft angle, as the ignitions occur at equidistant intervals in the diagram. Very high impacts occur when the engine stops at TDC or when the engine does not reach TDC at all due to the retroactive effect of the secondary flywheel. In the latter case, reverse combustions are produced with extreme impacts. The aim must be to anticipate this situation and disable the injection process in time. Until now, fixed speed limits have been implemented in the control system to disable the process, but Figure 13 shows that it is advisable to use an additional gradient-based limit. If a straight line is drawn in this diagram through the two 
previous ignition or injection points just before TDC, as shown in Figure 13, it is immediately apparent that the engine will stop at approx. $0 \mathrm{rpm}$ when it reaches the next TDC. This causes the high impacts afterwards.

The last ignition or injection were therefore not only useless - the engine was at a standstill afterwards - they also damaged the DMF and it would have been better for them to have been disabled by the engine control unit. These types of problems can now be identified early on in the project using simulations. By them, it is apparent that even a small difference of $10 \mathrm{~ms}$ in the engagement time can cause a tremendous difference in the impact level (Figure 14).

This finding also matches the large variations observed time and again in road tests. Statistical analysis is therefore essential, and can be conducted by means of simulations using a well-calibrated model (Figure 15).

These simulations then form the basis for estimating field quality. During this process, the behaviour of multiple drivers is calculated using Monte Carlo methods (rolling the dice for impact levels) in conjunction with the $\mathrm{S} / \mathrm{N}$ curves of the arc spring and the regularity of occurrence. It is possible to evaluate the software using the simulation

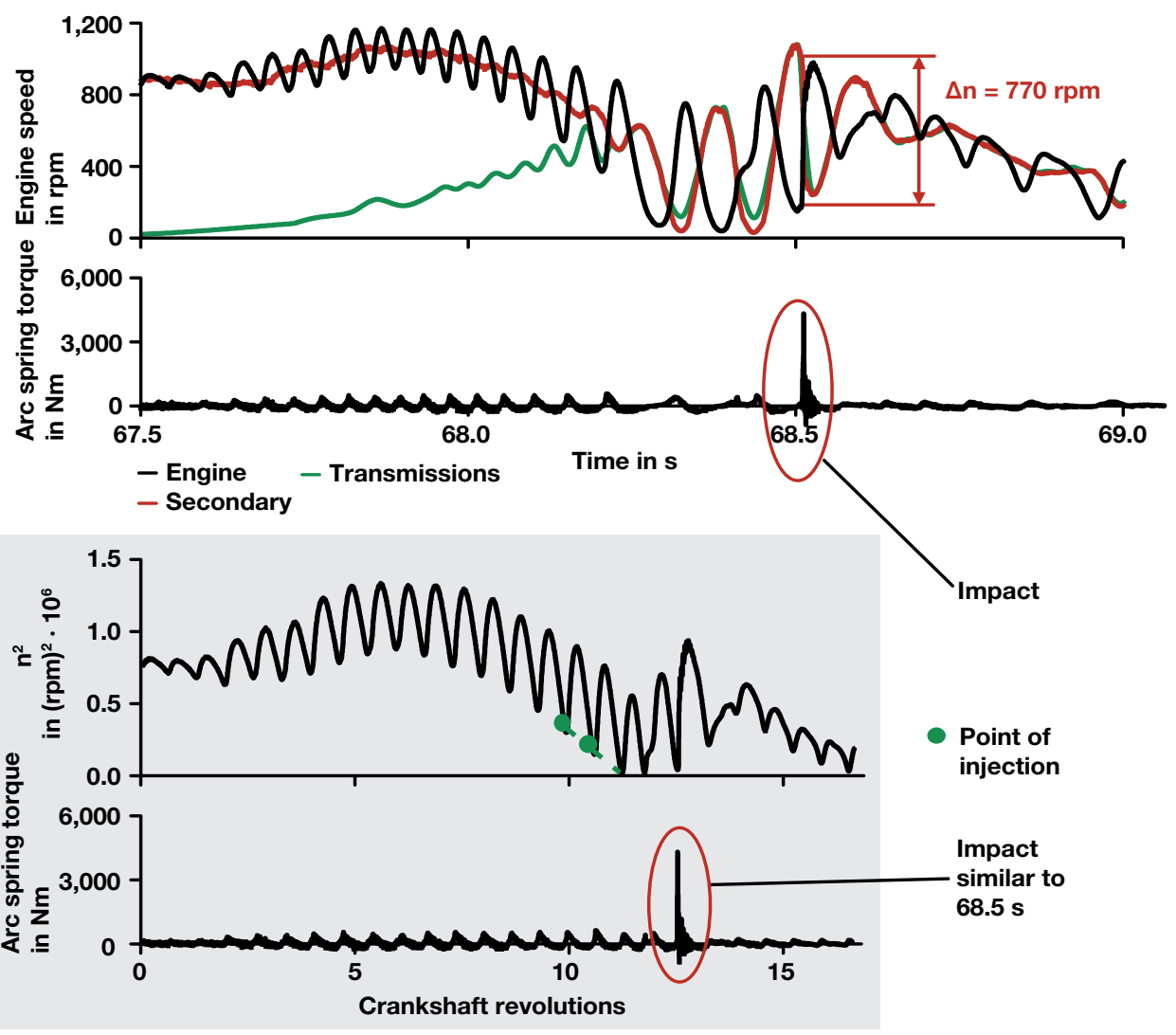

Figure 13 Impact when stalling 

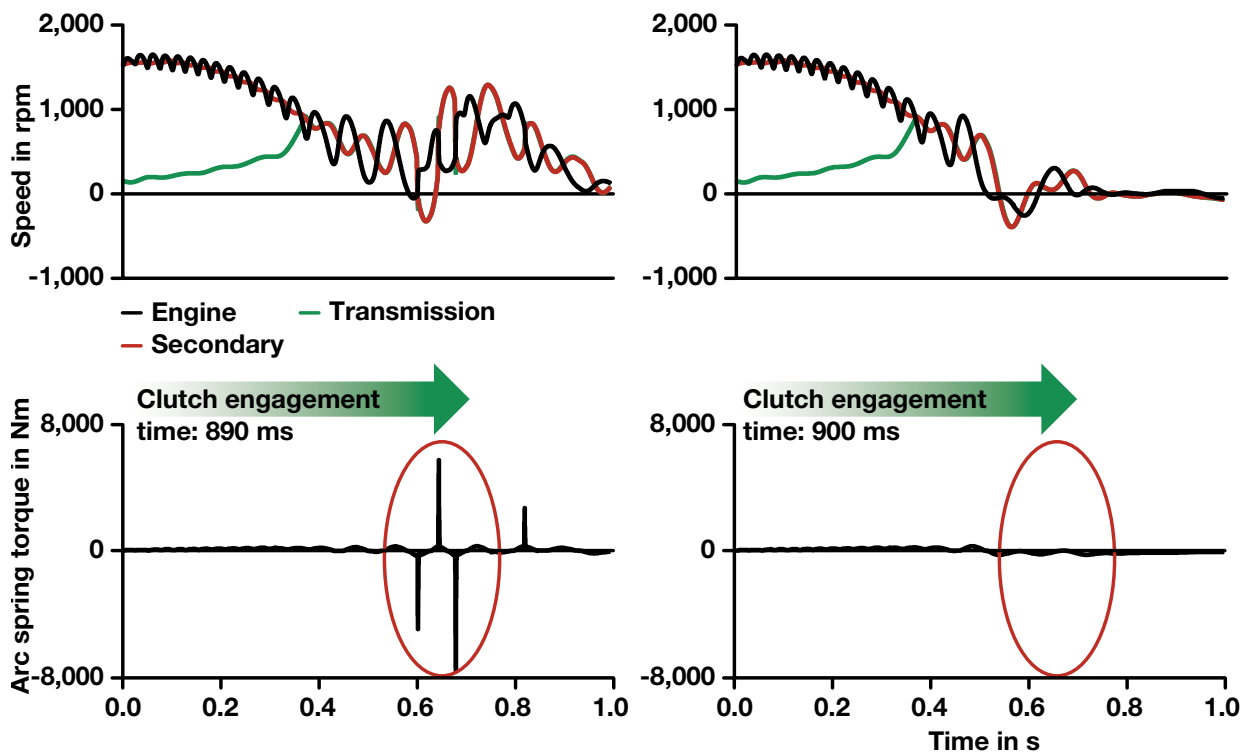

Figure 14 Influence of clutch engagement time on the impact level in stalling simulations

by integrating the software parameters. It is important to trigger necessary software adjustments early on in the project, preferably at the start of the project, as testing of software changes is extremely time-consuming. The engine control unit should also prevent the engine being restarted by the continuing motion of the vehicle after stall-

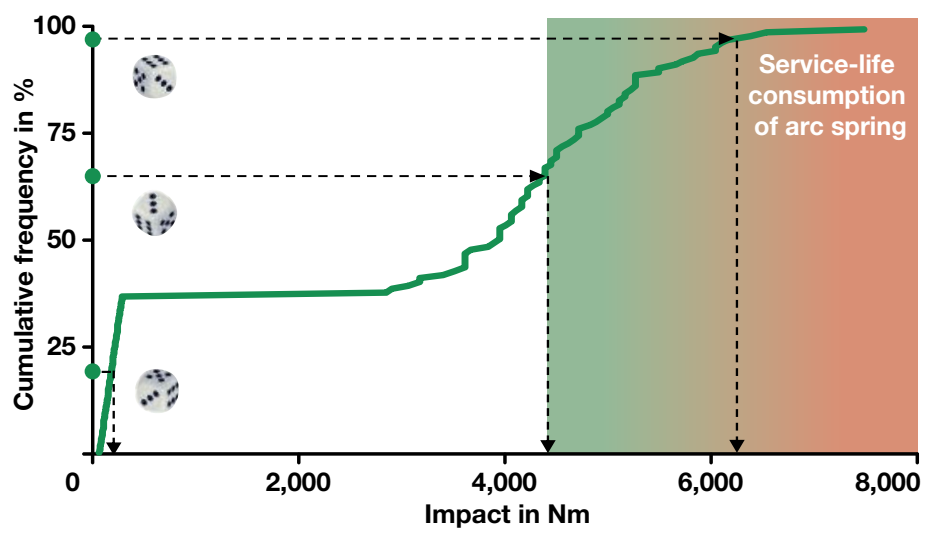

ing as this causes speed ratios and impacts that are difficult to control.

\section{The High Capacity spring}

It is difficult to develop an active engine control unit strategy that can prevent impacts entirely for all operating conditions and combinations of parameters. Therefore, the remaining impacts must be intercepted by an increased robustness of the DMF. This is where the High Capacity spring ( $\mathrm{HC}$ spring) can play a vital role (Figure 16).

Figure 15 Cumulative frequency of impacts during stalling and illustration of the Monte Carlo method 


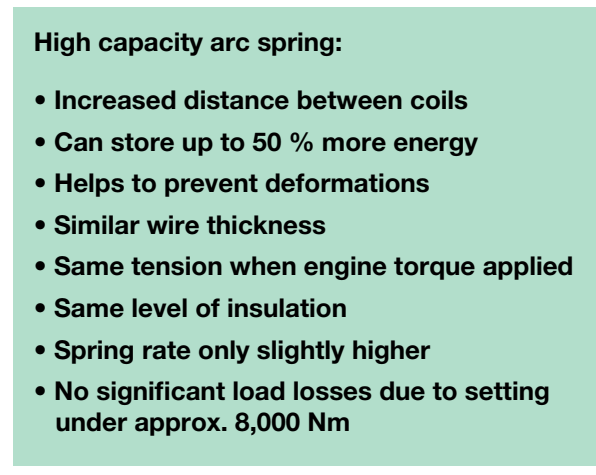

Figure 16 High capacity spring (HC spring)

The basic idea is to considerably increase the torque capacity of the arc spring and therefore absorb approx. $30 \%$ to $50 \%$ more energy in the characteristic curve, without hitting the end stop. Figure 17 shows the end of a start-up procedure, in which high clutch torque results in the damper striking the end stop.

The higher torque capacity of the $\mathrm{HC}$ spring is achieved by an increased distance between the coils and largely absorbs the high clutch torque. Wire thickness is kept approximately the same, so that the stress exerted on the springs by engine torque, and thus the service life, remains unchanged. As the distance between the coils increases as a consequence of the concept, fewer coils can be accommodated in the same space. The nominal spring rate therefore increases slightly. This affects starting behaviour to a small extent, but not drive characteristics. This is because the rear coils are disabled in drive mode as a result of the friction caused by centrifugal force. The shortened spring consequently has absolutely no effect on reducing the number of active coils.

Fatigue strength is not an issue for small impacts as impacts are relatively rare typically fewer than 1,000 load cycles over the vehicle's service life. The determined

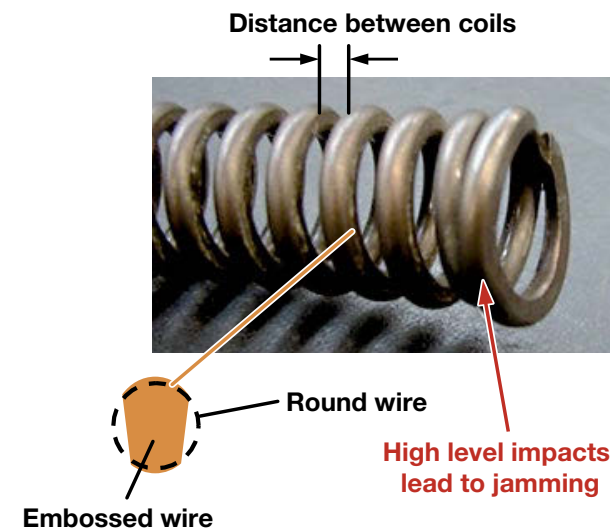

service life for small impacts is more than an order of magnitude greater. However, if higher impacts does happen to act on the $\mathrm{HC}$ spring despite its capability of absorbing energy, flattened coils can absorb the difference without serious crushing. LuK has used flattened coils successfully on standard springs for quite some time now. As $\mathrm{HC}$ springs have a significantly higher torque capacity than standard springs, set $\mathrm{HC}$ springs can still safely absorb the engine torque. Overall, HC springs yield huge benefits for the DMF in terms of robustness without compromising torsion isolation.

\section{Summary}

The evolution of the centrifugal pendulumtype absorber in conjunction with overall damper tuning improved the isolation achieved by DMFs to such an extent that it can also cope with higher engine torques and cover today's three-cylinder and even twin-cylinder engines. Furthermore, they still have further potential, as regard to isolation, for dealing with the expected further 


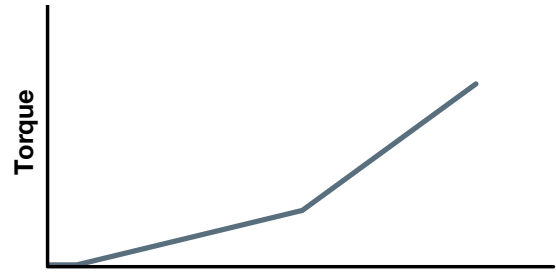

DMF torsion angle - Standard production spring

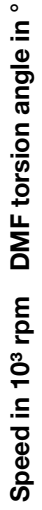

들

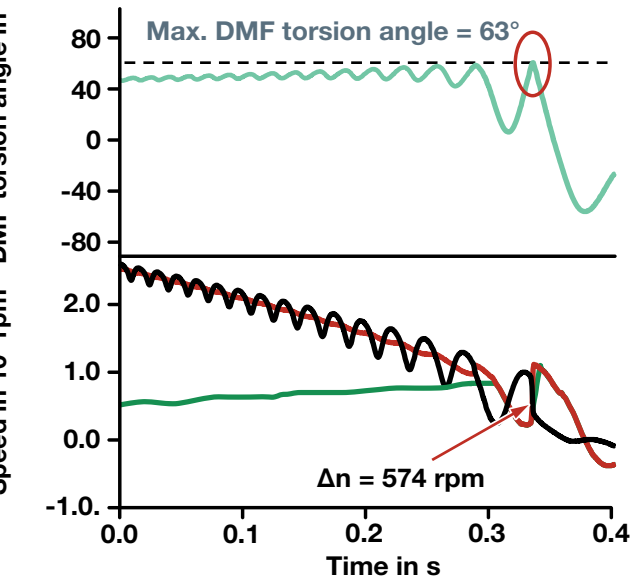

- Engine - Transmission - Secondary

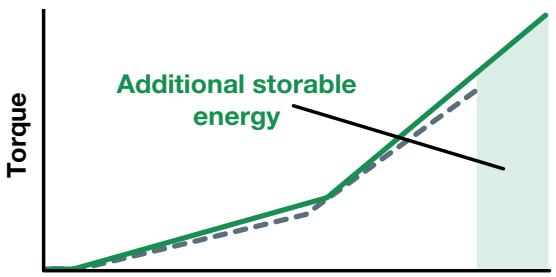

DMF torsion angle - HC-Spring

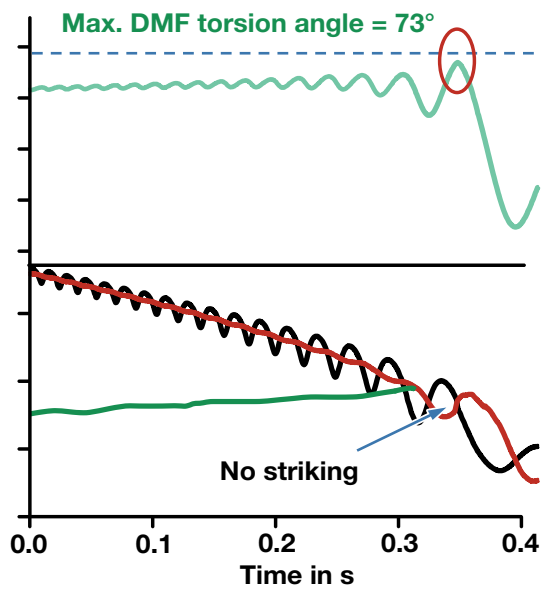

Figure 17 Influence of the $\mathrm{HC}$ spring when driving off

increase of engine torque from idle speed upwards. However, close interaction between powertrain design and damper concept is absolutely essential if this potential is to be achieved.

Locating the centrifugal pendulum-type absorber on the clutch disc succeeded in providing a long-awaited solution halfway between a simply damped clutch disc and a DMF. For trucks, arranging the CPA on the single mass flywheel also leads to reduced strain on the gearbox and the belt drive. Impact situations can be managed through early optimisation of the engine control unit and the use of Hlgh Capacity springs. No additional protective measures must then be implemented in the DMF; the system comprising DMF and centrifugal pendulum- type absorber can be designed specifically for maximum isolation.

\section{Literature}

[1] Kroll, J.; Kooy, A.; Seebacher, R.: Land in sight? $9^{\text {th }}$ Schaeffler Symposium, 2010,

[2] Kooy, A.; Gillmann, A.; Jaeckel, J.; Bosse, M.: DMF - Nothing new? $7^{\text {th }}$ LuK Symposium, 2002

[3] Reik, W.: Torsional vibration isolation in the powertrain. $4^{\text {th }}$ LuK Symposium, 1990

[4] Schneider, M. et al.: The Clutch Comfort Portfolio: From a supplier's product to an equipment criterion. 10 ${ }^{\text {th }}$ Schaeffler Symposium, 2014

Open Access. This chapter is distributed under the terms of the Creative Commons Attribution Noncommercial License, which permits any noncommercial use, distribution, and reproduction in any medium, provided the original author(s) and source are credited. 